\title{
Research on On-line Monitoring Device of Circulation in High Voltage Cable Metal Sheath
}

\author{
Tan deqing $^{1}$, Bai han ${ }^{1}$, Sun keqiang ${ }^{1}$, Wang zhenhao ${ }^{2}$ \\ ${ }^{1}$ State Grid LiaoNing Electric Power Company Limited Shenyang Power Supply Company, Shenyang, LiaoNing Shenyang \\ 110811, China \\ ${ }^{2}$ Northeast Dianli University, Jilin Jilin 132012, China
}

\begin{abstract}
We have designed an integrated high voltage cable metal sheath circulation online monitoring terminal. The open-type current sensor, the circuit of data acquisition and the circuit transceiver of radiofrequency wireless has been researched and developed, real-time online monitor the changes of high voltage cable metal sheath circulation, monitor the data is transmitted to the management system of the data analysis through wireless. It has been installed on the site and tested.
\end{abstract}

\section{Introduction}

High voltage cable due to its structure using single core structure would inevitably lead to the induced voltage appears on the metal sheath. If the grounding mode is improper, the induced voltage will form a large induced current on the metal sheath. This will bring two major hazards on cable transmission line. One is the ability of the cable's transmission of electricity reduce greatly (about one-third or so), the other is leading to metal sheathed heating to lower the main insulation and shorten the normal operating life of the cable. Therefore, the high voltage cable metal sheath must adopt the correct grounding mode to ensure that circulation is minimized.

Adopting cross interconnected grounding method can effectively reduce the circulation, the relations between value of circulating current and the segmented uniformity of cable are great, at the same time, cable laying uses the arrangement of regular triangle as far as possible, if the position of three-phase asymmetric, even if the piecewise uniform, circulation would be generated in metal sheath.

Therefore, in order to facilitate the safe operation of city power grid it is timely monitor the insulation of cable and the condition of the insulation of outer sheath and locate and repair the damage of the sheath.

On-line monitoring device of circulation in high voltage cable metal sheath that is studied in this paper could online monitor the changes of cable's circulation real-time, is going to warn and alarm early and suggests operator to take the necessary measures to ensure the safe operation of the cable.

\section{On-line monitoring terminal of circulation of the metal sheath}

Monitoring terminal is mainly composed of a microcontroller circuit 、 $\mathrm{AD}$ acquiring circuits 、 $\mathrm{RF}$ transceiver circuits and a power circuit that uses induction to take electricity, the ground line of three-phase metal sheath passed the type of opening current transformer, the signal is organized and transformed and then it is send to transform in $\mathrm{AD}$, then they are going to data processing in CPU, the measured data is transmitted through wireless radiofrequency circuit to remote relay device which can collect the monitoring data. The general structure is

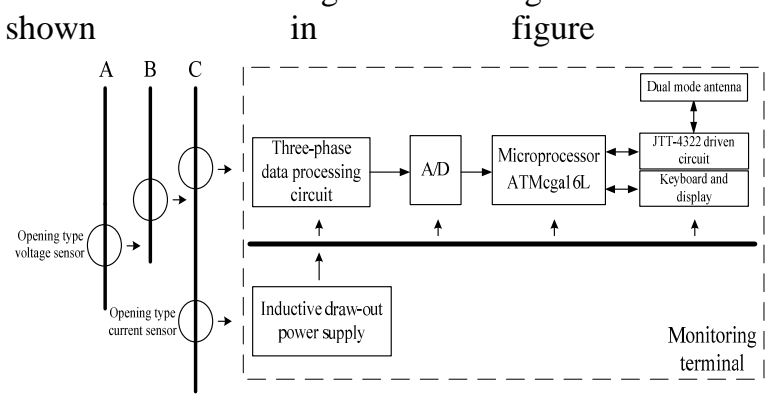

Figure 1. Monitoring terminal's structural block diagram

\subsection{The design of the microprocessor circuit}

Microcontrollers adopt TI's MSP430 MCU MSP430F2131.The working voltage of MSP430F2131 is 1.8 to $3.6 \mathrm{~V}$, the current is around $200 \mathrm{uA}$ in normal working conditions, the current is only $0.7 \mathrm{uA}$ under the condition of dormancy. It has a unique advantage that uses battery to supply electricity could achieve ultra-low-power consumption. The microprocessor circuit is shown in figure 2.

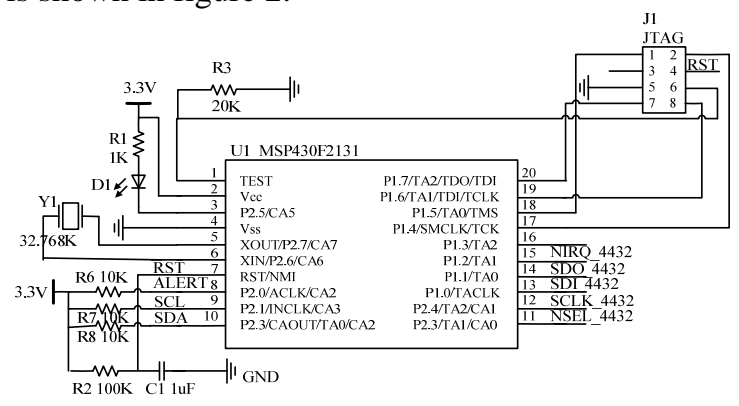

Figure 2. Microprocessor circuit

\subsection{The design of the data acquisition circuit}

The opening type current transformer outputs current signals and then they are sent to be organized in monitoring terminal, through the resistance, they are turned into voltage signals, then they are finished in launched grade follower, they are sent to AD circuit, so they are converted to digital, then send them to the CPU to calculate. As shown in figure 3 . 


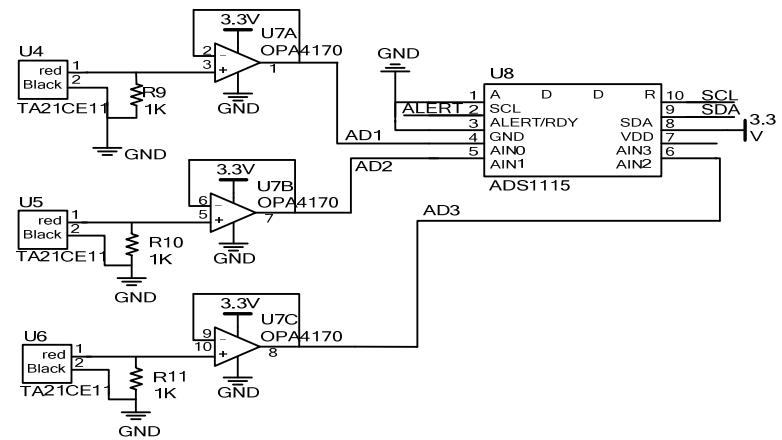

Figure 3. Data acquisition circuit

\subsection{The design of monitoring terminal's wireless radiofrequency communication circuit}

Communication circuit is based on high-performance wireless radiofrequency chip JTT4432, works in 433/470/915 MHZ in general ISM frequency band, it has a modulator and a demodulator, it can package automatically when it is sent, it can check CRC automatically and match address automatically when it is received, after finishing sending and receiving, its NIRQ interrupt pin will automatically be set to high level to indicate the ending of sending or receiving, Si4432 provides a SPI interface to the application of the controller and the speed is determined by the microcontroller itself, thus the programming is very convenient. Its power consumption is very low, the current is $85 \mathrm{~mA}$ when it is emitted by $20 \mathrm{dBm}$ output power, the current is $15 \mathrm{~mA}$ in receive mode and the standby current is only $2.5 \mathrm{uA}$. JTT 4432 has four working modes in total, they are losing electricity and SPI programming mode 、stand-by and SPI programming mode、 the reception mode、the transmission mode, the switching of its working mode is achieved through the configuration of register $07 \mathrm{H}$. Circuit is shown in figure 4.

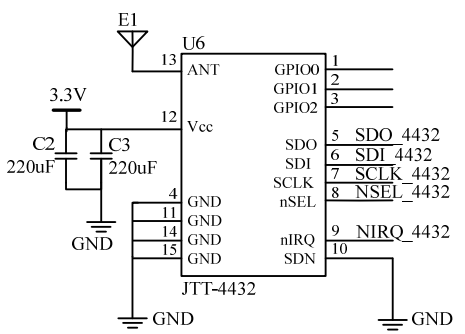

Figure 4. Wireless radiofrequency circuit

\subsection{The power supply using induction taking energy}

Monitoring terminal is installed in the cable grounding box, the voltage of the cable is high and it is fully enclosed, we can't acquire the power supply directly. In this paper using metal sheath grounding line can get the power supply by means of induction taking energy. Getting the voltage by the opening type current transformer after rectifying and filtering access to the energy bleed-off circuit and limit voltage when the current is high, it is outputted after DC conversion, we added the super capacitor in the circuit design, avoiding voltage dropping when instantaneous power is large. The circuit structure is shown in figure 5 .

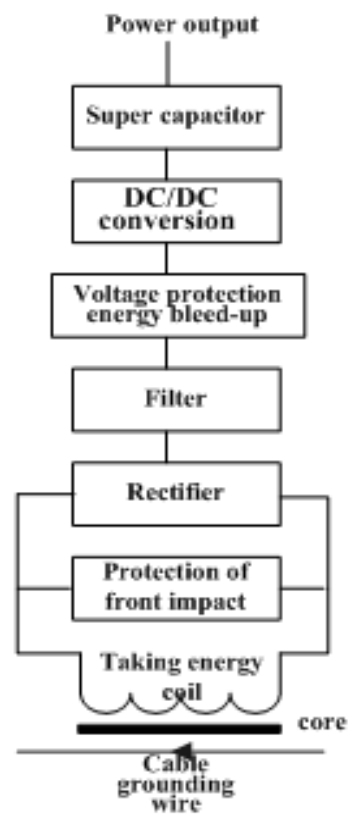

Figure 5. The structure of induction taking energy

\section{The operating on site}

Monitoring terminal is installed and debugging and running in a certain power supply company's $22 \mathrm{KV}$ cable lines, the telemetry of the remote cable equipment for many times in the monitoring center. According to the comparative analysis, all monitoring data is accurate and data is transferred smoothly, the indicators accord with technical requirements. Monitoring terminal and data are shown in figure 6. 

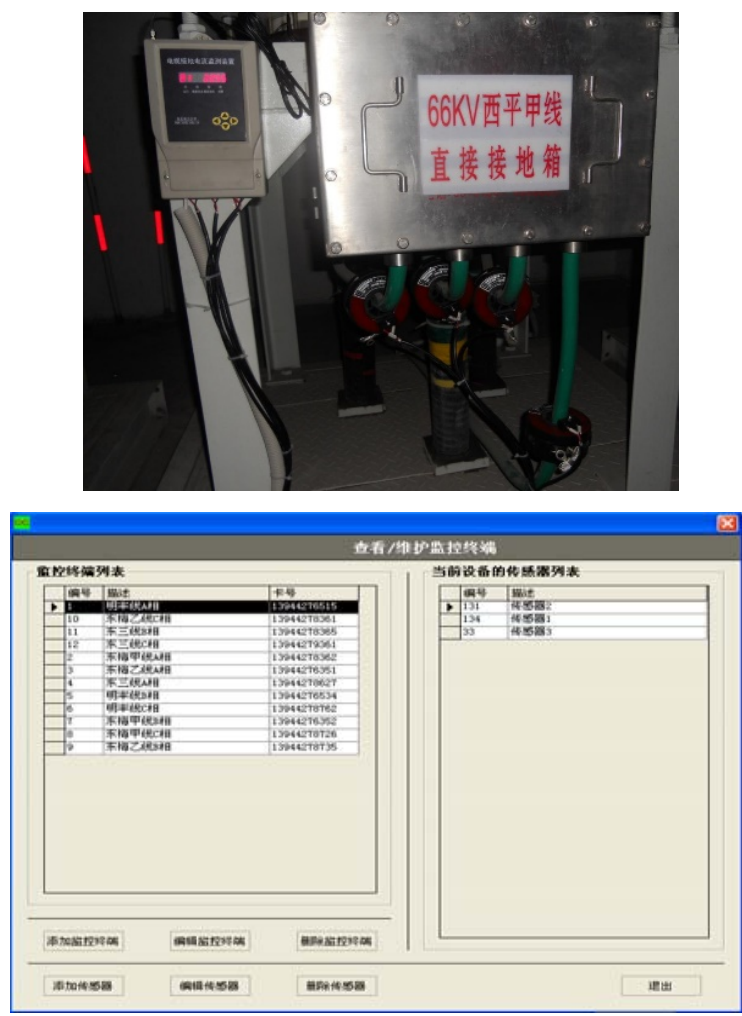

Figure 6. Monitoring device was installed in the field and monitoring data

\section{Conclusion}

In this paper, we have designed an integrated on-line monitoring terminal which can monitor the metal sheath circulation of the high voltage cable. Data acquisition, induction taking power, wireless communications are integrated, a detailed plan of design and the circuit are given. Tested in the laboratory and practical on-site, the indicators have reached the requirements of design, it has been applied on site to solve the current situation that the operators on site have to do the artificial test on regular intervals and the running effect is very good.

\section{Reference}

[1] DONG Xiaobing, CAI Jun, JIANG Xiuchen, et al. On-line monitoring techniques for $10 \sim 35 \mathrm{kV}$ XLPE cable. J. Electric Power Automation Equipment, 2005, 25 (9)

[2] 2. WEI Shurong, MA Hongzhong, WANG Donghai. The variation and compensation of induced voltage in the metal shield for the power cable renovation. J. Electric Wire \& Cable, 2004 (4)

[3] SUN Hongmei, LUO Longfu, LU Jiazheng, et al. $110 / 220 \mathrm{kV}$ XLPE cable insulation on-line monitoring technique. J. High Voltage Engineering, 2004, 30 (1)

[4] GU Huang-jin, QIU Qi-wei CHEN, Xiao-ping, et al Practicality analysis of $500 \mathrm{kV}$ cable online partial discharge monitoring system. J. East China Electric Power, 2014 (4) , 0788-0790.

[5] CHEN Qiao-yong, WEN Xi-shan, WU Gui fang, et al. Research on DC superposition method of cross-linked polyethylene power cable. J. Insulating Materials, 2002, 35 (6)

[6] ZHU Xiao-hui, DU Bo-xue, ZHOU Feng-zheng, et al. Research of high voltage XLPE cable online monitoring and detection technology. J. Insulation Material, 2009, 42 (5) 